\title{
Laser surface melting of stainless steel anodes for reduced hydrogen outgassing
}

\author{
D. Gortat ${ }^{\mathrm{a}, *}$, P.T. Murray ${ }^{\mathrm{b}}$, S.B. Fairchild ${ }^{\mathrm{c}}$, M. Sparkes ${ }^{\mathrm{a}}$, T.C. Back ${ }^{\mathrm{b}}$, G.J. Gruen ${ }^{\mathrm{b}}$, M.M. Cahay ${ }^{\mathrm{d}}$, \\ N.P. Lockwood ${ }^{\mathrm{e}}$, W. O’Neill ${ }^{\text {a }}$ \\ a Institute for Manufacturing, University of Cambridge, 17 Charles Babbage Road, Cambridge CB3 OFS, UK \\ ${ }^{\mathrm{b}}$ Research Institute, University of Dayton, Dayton, OH 45469, USA \\ ' Air Force Research Laboratory, WPAFB, OH 45433, USA \\ ${ }^{\mathrm{d}}$ Spintronics and Vacuum Nanoelectronics Laboratory, University of Cincinnati, Cincinnati, OH 45221, USA \\ e Directed Energy Directorate, Air Force Research Laboratory, Kirtland, AFB, NM 87117, USA
}

\section{A R T I C L E I N F O}

Article history:

Received 1 June 2016

Received in revised form 11 December 2016

Accepted 28 December 2016

Available online 29 December 2016

\section{Keywords:}

Grain boundaries

Laser processing

Metals and alloys

Vacuum electronics

Hydrogen

Outgassing

\begin{abstract}
A B S T R A C T
Anodes of 304 stainless steel have been processed by a continuous wave $\mathrm{Yb}$ fiber laser with a wavelength of $1.064 \mu \mathrm{m}$ and subjected to $50 \mathrm{keV}$ electron bombardment in order to determine the extent to which hydrogen outgassing is reduced by the laser surface melting treatment. The results show a reduction in outgassing, by approximately a factor of four compared to that from untreated stainless steel. This is attributed to a reduction in the number of grain boundaries which serve as trapping sites for hydrogen in stainless steel. Such laser treated anodes do not require post-processing to preserve the benefits of the treatment and are excellent candidates for use in high power source devices.

Crown Copyright @ 2016 Published by Elsevier B.V. This is an open access article under the CC BY license
\end{abstract} (http://creativecommons.org/licenses/by/4.0/).

\section{Introduction}

High Power Source (HPS) devices are used in numerous applications including vacuum electronics [1-3], particle acceleration $[4,5]$, and microwave generation $[2,6]$. Stable, long term HPS operation is presently constrained by pulse shortening due to plasma formation in the anode-cathode gap region. Plasma is formed through the interaction of secondary electrons and gas molecules (primarily hydrogen), both of which are released by the impact of high energy electrons at the anode surface. Metals with relatively low hydrogen outgassing rates, such as austenitic stainless steel (SS) [7], are the most commonly used materials for vacuum applications [7]. To further reduce outgassing in such metals, several treatments have been proven effective including baking [8$12]$, vacuum baking $[8,11,13,14]$, polishing $[8,14]$, and surface treatments to create oxide or other protective surface films. Electropolishing has been the method of choice $[8,11,15]$ since it both reduces the surface roughness of anode materials and creates an oxide layer that further reduces hydrogen outgassing. However, electropolishing may also introduce hydrogen and other contami-

\footnotetext{
* Corresponding author.

E-mail address: dg458@cam.ac.uk (D. Gortat).
}

nants into the surface layers in significant quantity [15] and may necessitate an additional bake to thoroughly degas the surface [8].

The purpose of the work described here was to determine the feasibility of reducing hydrogen outgassing by processing anodes of 304 SS by laser surface melting (LSM). The LSM processing technique entails irradiating a sample with the output of a high energy continuous laser beam, thereby causing melting, flow and resolidification of the material as the laser beam is scanned across the sample surface. This process has the potential of reducing outgassing by forming a more crystalline layer (with fewer grain boundaries), thereby reducing the number of potential sites that can trap hydrogen in the metal [16]. When compared to more conventional processing techniques, such as electropolishing, the LSM process introduces significantly fewer contaminants (especially hydrogen) into the anode surface. We show here that LSM processing of 304SS does indeed lead to reduced $\mathrm{H}_{2}$ outgassing during electron bombardment.

\section{Experimental}

Samples of 304SS were irradiated at normal incidence by a nonpolarized Continuous Wave (CW) SPI ${ }^{\mathrm{IM}} \mathrm{G} 3 \mathrm{Yb}$ fiber laser $\left(\mathrm{M}^{2}=2\right.$, input beam diameter $4.3 \mathrm{~mm}$ ), with a wavelength of $1.064 \mu \mathrm{m}$, 
maximum output power of $20 \mathrm{~W}$ and nominal spot size of $39.4 \mu \mathrm{m}$. The treatment was carried out at atmospheric pressure under constant $\mathrm{N}_{2}$ flow into the capped stage $\left(\mathrm{O}_{2}\right.$ levels $\left.<0.2 \%\right)$, as shown in Fig. 1. The beam expander telescope (BET) supplied an input beam of $4.3 \mathrm{~mm}$ in diameter to the laser head.

The lens used in the laser setup was a Jenoptic ${ }^{\mathrm{TM}}$ fused silica lens with focal length of $125 \mathrm{~mm}$. For patterning the sample, a bidirectional raster scan was applied with a line separation of $30 \mu \mathrm{m}$ and irradiated with a average laser energy density (ED) of $13.54 \mathrm{~kJ} / \mathrm{cm}^{2}$. Additional details of the LSM processing can be found elsewhere [17]. An Olympus BX51 ${ }^{\mathrm{TM}}$ optical microscope with JENOPTIC ${ }^{\mathrm{TM}}$ ProgResC10+CCD camera was used to obtain images and depth measurements of the treated samples. For the depth measurements, the SS samples were cut along the laser-scanning track and mechanically polished using standard metallographic techniques. The samples were chemically etched in SS micro-etchant, chemical composition $10 \mathrm{~g} \mathrm{FeCl}_{3} 30 \mathrm{ml} \mathrm{HCl} 120 \mathrm{ml}$ water, at room temperature to reveal the general microstructure. Scanning electron microscopy (SEM) images were acquired with a $\mathrm{FEI}^{\mathrm{TM}}$ Quanta 3D system equipped with a field emission gun (FEG). Microstructural characterization was conducted with the help of focused ion beam (FIB) microscopy and Philips XL30 SEM with FEG in secondary electron mode to obtain orientation maps. HKLTango ${ }^{\mathrm{TM}}$ software was used to quantify the grains and grain boundaries (GBs). GBs were categorized in two groups, special $\left(3<\sum \leqslant 29\right)$ and random $\left(29<\sum \leqslant 49\right)$, where $\sum$ is the reciprocal of the fraction of the common lattice sites (CSL) from each grain at the boundary [18]. More restrictive Palumbo-Aust criterion [19] is used to determine the $\sum$ number.

Outgassing characterization was carried out by bombarding at normal incidence the SS samples with the focused output of a $50 \mathrm{keV}$ electron beam with a spot size of $1.6 \mathrm{~mm}$ in diameter (determined by measuring the size of a hole formed in a thin $\mathrm{Ni}$ foil under conditions identical to those used for the present work) with 60 s duration current pulse, and recording the time evolution during the pulse of the $\mathrm{H}_{2}$ signal with a residual gas analyzer situated 45 degrees from the surface normal. The electron current density at the sample surface was approximately $16.4 \mathrm{~mA} / \mathrm{cm}^{2}$, and the base pressure was $5 \times 10^{-10}$ Torr.

\section{Results \& discussion}

In order to form samples for depth characterization and outgassing evaluation, the SS samples were processed by raster scanning the laser beam across the surface in a uniform pattern. Shown in Fig. $2 a$ is an SEM image of the raster scanned surface of the SS sample, tilted at 45 degrees to the electron beam column; the LSM-treated and untreated areas are visible. The individual laser tracks can be seen as can the ripples within each track that are ori-

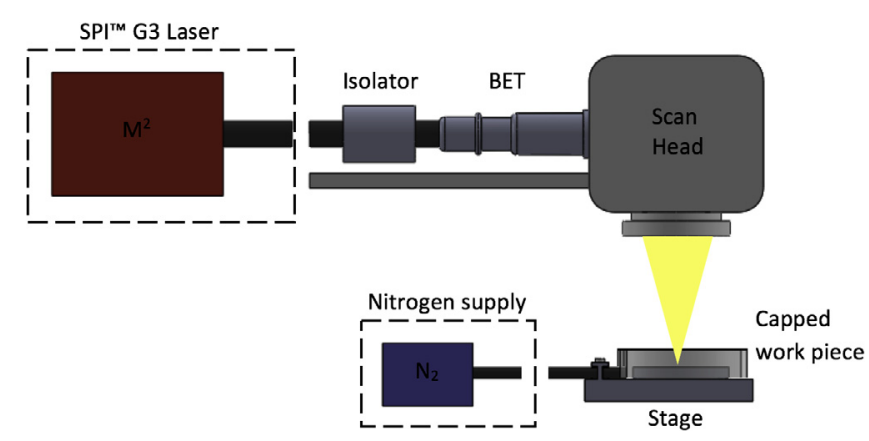

Fig. 1. Laser processing workstation showing the $\mathrm{Yb}$ fiber laser, Isolator for preventing unwanted feedback into the laser, BET, nitrogen supply and sample stage. ented in the direction of the laser beam scan; a phenomenon in CW laser melting commonly observed at high laser ED values [20]. Processing with lower ED tends to avoid ripple formation in the irradiated area but also reduces grain growth in the lattice of the sample. There are also small, bump-like formations seen within the treated area. Note that the features extend above the plane of the laser treated area and occur near the overlap between adjacent laser scan lines. These features represent areas of the SS surface morphology that were incompletely melted by LSM and most likely result from the specific choice of scan parameters (laser spot size and degree of overlap between adjacent scan lines) for the results shown here. These features can be minimized by a different choice of initial scan parameters or by subsequent laser passes over the sample. Shown in Fig. $2 \mathrm{~b}$ is a longitudinal cross-section (optical) view of the treated sample, where the treated region is comprised of an LSM zone (LSMZ) and heat-affected zone (HAZ). The depth of the LSMZ is approximately $9.7 \mu \mathrm{m}$.

Shown in Fig. 3a and b are FIB images of the untreated and treated samples, respectively, demonstrating the microstructural changes induced by the laser radiation. It can be observed that the grains in the laser treated area, Fig. $3 \mathrm{~b}$, are elongated in the direction of the laser scan and have increased in size as a result of laser processing, as detailed in Table 1. The total number of grains was reduced from 1020 to 617 per $0.12 \mathrm{~mm}^{2}$ unit volume. This transformation has the net effect of reducing the number of special and random GBs in the laser-treated volume and supports the outgassing data showing less $\mathrm{H}_{2}$ released from such samples. Table 1 suggests that it is the grain boundary character distribution, i.e., the spectrum of misorientations and inclinations which is changed as a result of laser melting. Increasing further the grain size would entail extending the surface cooling time [21]. In fact, as shown in Fig. 2, during laser melting only the thin surface layer is melted which then rapidly solidifies due to the high heat outflow toward the solid substrate. Therefore, we believe that in our case the spectrum of misorientations and inclinations corresponds to a temperature close to the melting temperature $T_{m}$. However, it is well known that the equilibrium grain boundary character distribution changes significantly with temperature [22,23]. For instance, in conventional solidification, the temperature actually decreases slowly, and the spectrum of grain boundary misorientations and inclinations corresponds to a lower temperature of about $0.5 T_{m}$. To optimize the reduction of hydrogen outgassing from stainless steel anodes using the laser surface treatment reported here, a more thorough analysis would require a study of the grain boundary character distribution as a function of the laser spot size and energy density [22,23].

Shown in Fig. 4 are the outgassing results, which are presented as the change in $\mathrm{H}_{2}$ partial pressure (above baseline) with electron dose. Both curves exhibit an increase in $\mathrm{H}_{2}$ signal with electron dose. Note that the $\mathrm{H}_{2}$ signal for the untreated sample does not immediately rise with electron dose but instead shows an incubation period (extending from a dose of 0 to $\sim 3 \times 10^{18} \mathrm{~cm}^{-2}$ ) in which the $\mathrm{H}_{2}$ signal first increases gradually, after which there is a more rapid, nonlinear increase. Hydrogen is present within many metals, where it exists as atomic $H$ at defects such as GBs [16]; Hydrogen outgassing from metals occurs [24] through a series of steps consisting of (a) diffusion of atomic $\mathrm{H}$ to the metal surface, (b) recombination of atomic $\mathrm{H}$ at the surface and (c) desorption of nascent, molecular hydrogen $\mathrm{H}_{2}$ into the gas phase. The incubation period most likely is comprised of step (a) in which the majority of incident electron flux goes toward heating the sample and enhancing $\mathrm{H}$ atom diffusion to the surface. Only after the induction period is there sufficient atomic $\mathrm{H}$ at the surface that steps (b) and (c) proceed more efficiently and a larger desorbed $\mathrm{H}_{2}$ signal is detected. The data from the LSM-treated sample exhibits a smaller initial slope, suggesting a lower rate of diffusion of atomic $\mathrm{H}$ to the 

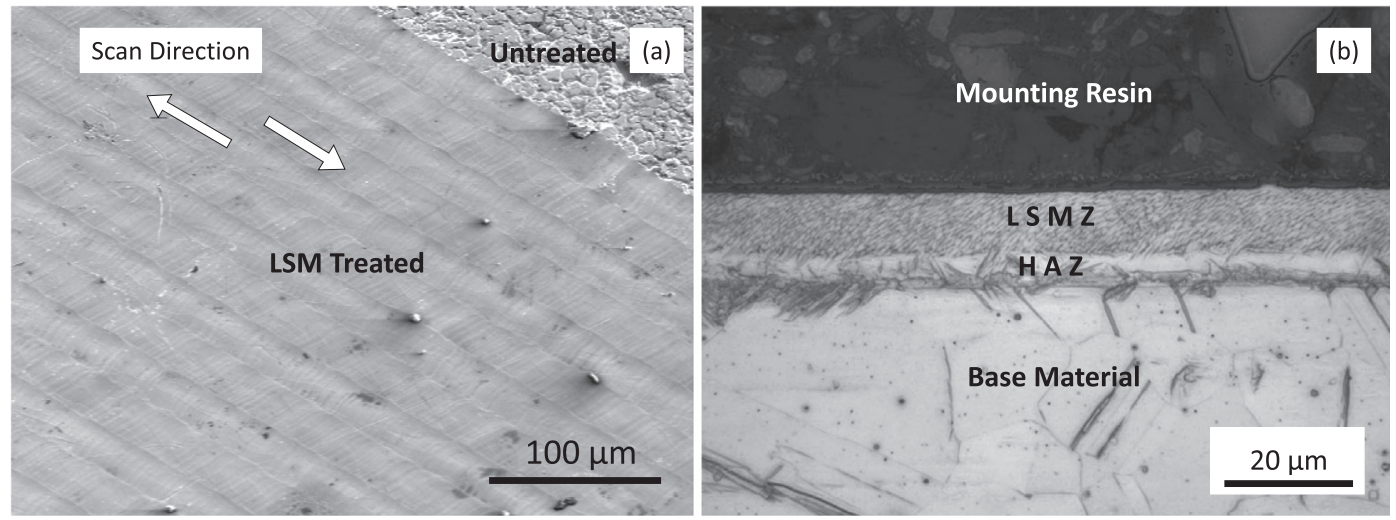

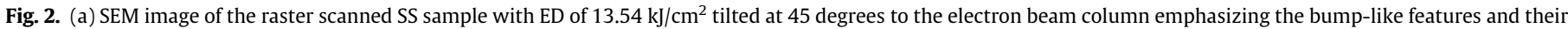

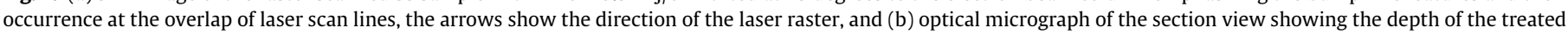
region.
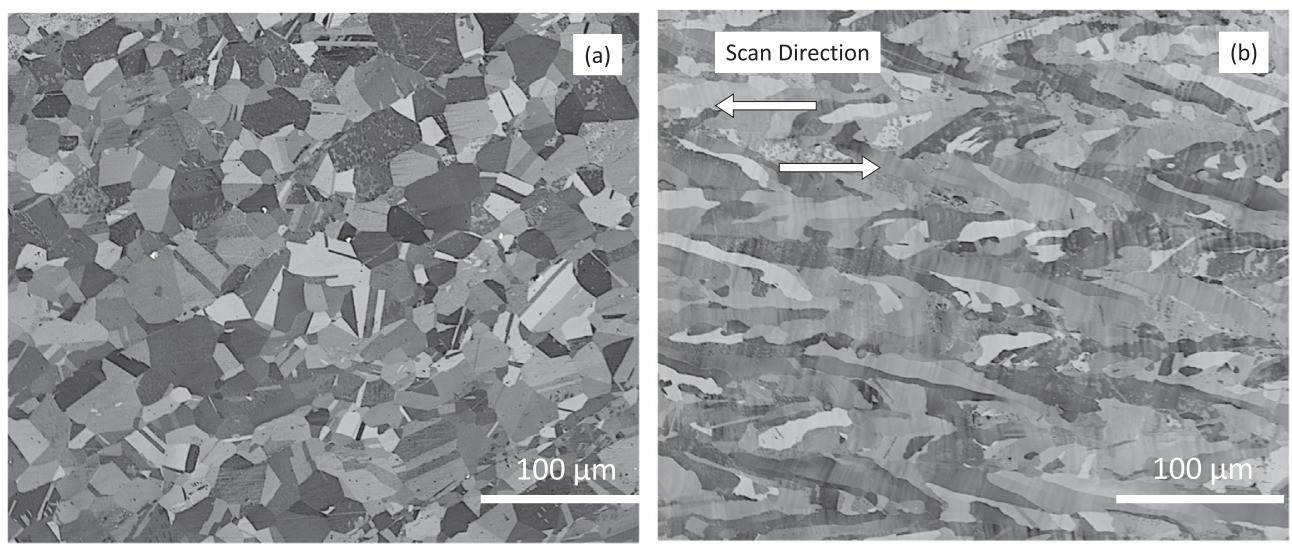

Fig. 3. FIB image showing the microstructure of the (a) untreated and (b) treated SS sample. The arrows show the direction of the laser raster.

Table 1

Grain boundary character distribution per $0.12 \mathrm{~mm}^{2}$ surface area.

\begin{tabular}{|c|c|c|c|c|c|c|}
\hline Position & $\Sigma 3$ & $\Sigma 9$ & $3<\sum \leqslant 29$ & $29<\sum \leqslant 49$ & Average grain size $(\mu \mathrm{m})$ & Average grain area $\left(\mu \mathrm{m}^{2}\right)$ \\
\hline Base material & 33.81 & 1.43 & 35.11 & 0.07 & 10.72 & 99.88 \\
\hline LSMZ & 15.39 & 0.82 & 16.66 & 0.05 & 14.12 & 201.59 \\
\hline
\end{tabular}

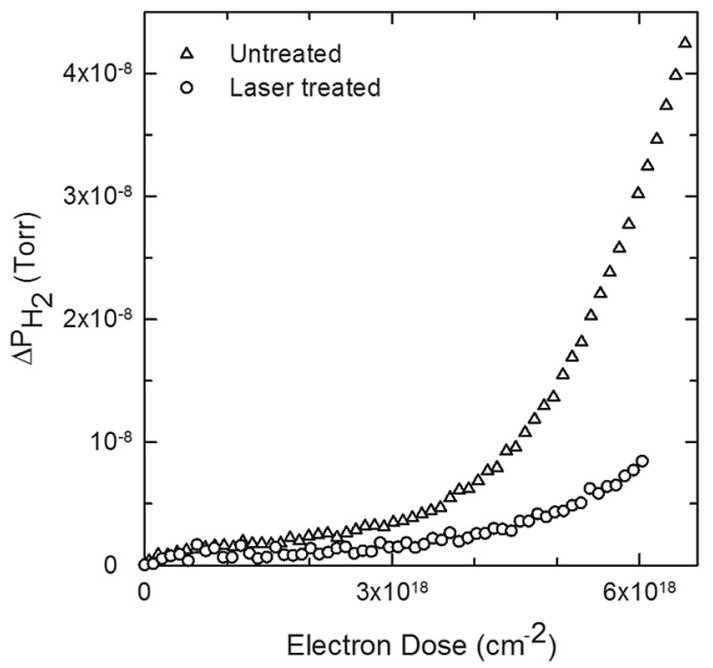

Fig. 4. Outgassing results showing the change in $\mathrm{H}_{2}$ partial pressure with electron dose during $60 \mathrm{~s}$ electron irradiation. surface because of the decreased number of GBs. The differences in these data sets are currently being investigated in more detail.

The data indicate that $\mathrm{H}_{2}$ outgassing from the LSM-treated sample was approximately a factor four less than that from the untreated SS sample, for the conditions employed here.

\section{Conclusions}

304SS treated with $\mathrm{CW}$, non-polarized $\mathrm{Yb}$ fiber laser radiation at an ED value of $13.54 \mathrm{~kJ} / \mathrm{cm}^{2}$ showed reduced hydrogen outgassing by a factor of $\sim 4$ at an electron dose of $6 \times 10^{18} \mathrm{~cm}^{-2}$, indicating the feasibility of the LSM process for reducing hydrogen outgassing from SS anodes. Such laser treated anodes do not require postprocessing to preserve the benefits of the treatment. The mechanism of suppression of hydrogen outgassing is caused by stimulating grain growth in the lattice of the specimen. Further studies are planned to maximize hydrogen outgassing reduction via grain boundary character distribution as a function of the laser spot size and energy density. 


\section{Acknowledgments}

Work supported by US Air Force contract FA8650-11-D-5401 at the Materials \& Manufacturing Directorate (AFRL/RXAP). The authors thank Lt Col Victor Putz of AFOSR/EOARD and Jason Marshall at AFOSR.D.G and M.S. wish to thank the EPSRC (EP/ K503241/1).

\section{References}

[1] K.E. Hackett, Directed Energy Applications for High Power Vacuum Electronics, Vac. Electron. Conf. 2006 Held Jointly with 2006 IEEE Int. Vac. Electron Sources, IEEE Int. 2006 (2006) 11-13.

[2] R.J. Barker, E. Schamiloglu, High-power microwave sources and technologies, Wiley-IEEE Press, 2001.

[3] J.H. Booske, R.J. Dobbs, C.D. Joye, C.L. Kory, G.R. Neil, G.S. Park, et al., Vacuum electronic high power terahertz sources, IEEE Trans. Terahertz Sci. Technol. 1 (2011) 54-75.

[4] S.D. Korovin, V.V. Rostov, S.D. Polevin, I.V. Pegel, E. Schamiloglu, M.I. Fuks, et al. Pulsed power-driven high-power microwave sources, Proc. IEEE 92 (2004) 1082-1094.

[5] D. Keefe, Research on high beam-current accelerators, Part, Accel. I (1981) 187-199.

[6] E. Schamiloglu, High power microwave sources and applications 2004 IEEE MTT-S Int. Microw. Symp. Dig. (IEEE Cat.No.04CH37535). 2 (2004) 999-1002.

[7] S. Avdiaj, B. Erjavec, Outgassing of hydrogen from a stainless steel vacuum chamber, Mater. Tehnol. 46 (2012) 161-167.

[8] P. Redhead, Extreme high vacuum, Proc. Cern Accel. Sch. Snekersten, Denmark, Cern Report, Ed. by S.Turn. (1999) 213-224.

[9] M. Bernardini, Air bake-out to reduce hydrogen outgassing from stainless steel, J. Vac. Sci. Technol. A Vacuum, Surfaces, Film 16 (1998) 188-193.
[10] K. Jousten, Thermal outgassing, Proc. Cern Accel. Sch. Snekersten, Denmark, Cern Report, Ed. by S. Turn. (1999) 111-124.

[11] M. Leich, Hydrogen outgassing of stainless steel, our present knowledge, in: Proc. 1st Vac. Symp., UK, 2010.

[12] P.A. Redhead, Hydrogen in Vacuum Systems: An Overview, in: AIP Conf. Proc., AIP, 2003. pp.243-254.

[13] L. Westerberg, B. Hjörvarsson, E. Wallén, A. Mathewson, Hydrogen content and outgassing of air-baked and vacuum-fired stainless steel, Vacuum 48 (1997) $771-773$.

[14] O.B. Malyshev, B.T. Hogan, M. Pendleton, Effect of surface polishing and vacuum firing on electron stimulated desorption from 316LN stainless steel, J. Vac. Sci. Technol. A Vacuum, Surfaces, Film 32 (2014), 051601.

[15] R.J. Reid, Cleaning for vacuum service, Proc. Cern Accel. Sch. Snekersten, Denmark, Cern Report, Ed. by S. Turn. (1999) 147.

[16] R.F. Berg, Hydrogen traps in the outgassing model of a stainless steel vacuum chamber, J. Vac. Sci. Technol. A Vacuum, Surfaces, Film 32 (2014), 031604.

[17] D. Gortat, Anode materials for high power microwave devices (M.S. thesis), Dept. Eng., Camb. Univ., Cambridge, UK, 2015.

[18] H. Grimmer, W. Bollmann, D.H. Warrington, Coincidence-site lattices and complete patter-shift lattices in cubic crystals, Acta Crystallogr A. 30 (1974) 197-207.

[19] G. Palumbo, K.T. Aust, E.M. Lehockey, U. Erb, P. Lin, On a more restrictive geometric criterion for special CSL Grain Boundaries, Scr. Mater. 38 (1998) $1685-1690$.

[20] A. Temmler, E. Willenborg, K. Wissenbach, Design surfaces by laser remelting, Phys. Procedia. 12 (2011) 419-430.

[21] N. Bidin, S.N. Ab Razak, ArF excimer laser annealing of polycrystalline silicon thin film, Cryst. - Sci. Technol., InTech (2012).

[22] L.S. Shvindlerman, B.B. Straumal, Regions of existence of special and nonspecial grain boundaries, Acta Metall. 33 (1985) 1735-1749.

[23] B.B. Straumal, S.A. Polyakov, E.J. Mittemeijer, Temperature influence on the faceting of $\Sigma 3$ and $\Sigma 9$ grain boundaries in Cu, Acta Mater. 54 (2006) 167-172.

[24] J.K. Gorman, W.R. Nardella, Hydrogen permeation through metals, Vacuum 12 (1962) 19-24. 